\title{
Shape Skeleton Classification Using Graph and Multi-scale Fractal Dimension
}

\author{
André R. Backes ${ }^{1}$ and Odemir M. Bruno ${ }^{2}$ \\ 1 Instituto de Ciências Matemáticas e de Computação (ICMC) \\ Universidade de São Paulo (USP) \\ Avenida do Trabalhador São-carlense, 400 \\ 13560-970 São Carlos SP Brazil \\ 2 Instituto de Física de São Carlos (IFSC) \\ Universidade de São Paulo (USP) \\ Avenida do Trabalhador São-carlense, 400 \\ 13560-970 São Carlos SP Brazil \\ backes@icmc.usp.br, \\ bruno@ifsc.usp.br
}

\begin{abstract}
This paper presents a novel approach to shape characterization, where a shape skeleton is modelled as a dynamic graph, and its complexity is evaluated in a dynamic evolution context. Descriptors achieved by using this approach show to be efficient in the characterization of different shape patterns with different variations in their structure (such as, occlusion, articulation and missing parts). Experiments using a generic set of shapes are presented as also a comparison with traditional shape analysis methods, such as Fourier descriptors, Curvature, Zernike moments and Bouligand-Minkowski. Although the reduced amount of information present in the shape skeleton, results show that the method is efficient for shape characterization tasks, overcoming the traditional approaches.
\end{abstract}

Keywords: graph, shape analysis, skeleton, multi-scale fractal dimension.

\section{Introduction}

Skeletons are useful representations of a shape. They are simpler structures, which preserve both topological and geometric properties of the shape. Their reduced information make easier recognition and analysis processes. Most of the methods for computing the skeleton use the Medial Axis Transform (MAT), originally proposed by Blum [1, 2]. The MAT is a set of center coordinates of circles interconnected that (i) have to be bi-tangents to the shape and (ii) be completely inside of the shape.

Literature presents various approaches for skeletons' recognition and analysis. These approaches can be grouped in the following categories: (i) metrics, it is based on measures of skeleton structure (such as, angles formed by lines, width and number of lines); (ii) fractal-based methods [3] ; (iii) signal analysis (such as 
Fourier and wavelet transform) and (iv) AI techniques, where the skeleton can be used directly as the input of a neural network [4, 5]. Although the diversity of methods and the reduced amount of information in the skeleton in comparison to the shape contour, the nature of the skeleton structure is not so easy to process, thus requiring special analysis.

This paper presents a graph-based approach to analyse the skeleton in terms of its complexity. Initially, the method was proposed for shape contours analysis [6]. In this paper, we apply it to the skeleton analysis. A comparative experiment is set in order to evaluate the performance of the method against traditional contour based methods. Results indicate the efficiency of the method in the skeleton analysis as its results overcomes the compared approaches.

\section{Shape Skeleton Modeled as a Graph}

Skeleton information computed from a shape can be easily interpreted as a graph (Figure 1). Consider the digital skeleton as a list of $N$ connected points $S$, $S=\left[s_{1}, s_{2}, \ldots, s_{N}\right]$. Each point $s_{i}=\left(x_{i}, y_{i}\right)$ represents the discreet coordinate of the point $i$ in the skeleton. Let $G=(V, E)$ be a graph, where $V$ is the set of vertices and $E$ is the set of links or edges. For each point $s_{i} \in S$, a vertex $v_{i} \in V$ is added in the graph $G$. A non-directed edge $e_{i j} \in E$ is built connecting each pair of vertices $\left(v_{i}, v_{j}\right)$, where the weight of this edge, $w_{i j}$, is given by the Euclidean distance between the respective points in the skeleton:

$$
w_{i j}=\sqrt{\left(x_{i}-x_{j}\right)^{2}+\left(y_{i}-y_{j}\right)^{2}} .
$$

Thus, the graph can be represented as a $N \times N$ matrix $W$

$$
W(i, j)=w_{i j}
$$

In order to avoid the influence of scale transformations in the graph, the weight of each edge is normalized into the interval $[0,1]$,

$$
W=\frac{W}{\max _{w_{i j} \in W}} .
$$

This graph modelling results in a graph where each vertex presents the same characteristic, i.e., a graph with regular behavior. This sort of graph has no relevant properties that can be used in its characterization. Thus, a transformation is applied over the graph in order to exploit the interaction between its structural and dynamical aspects [7, 8].

There are many approaches that can be used to perform this graph transformation, $\delta$. The use of a graph threshold is one of the most simple and straight approaches. Basically, a threshold $t$ is applied over the set of edges $E$, thus resulting in $E_{t}, E_{t} \subseteq E$, where

$$
E_{t}=\delta_{t}(E)=\left\{e_{i j} \in E \mid w_{i j} \leq t\right\}
$$




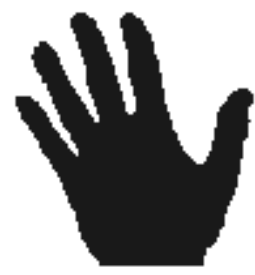

(a)

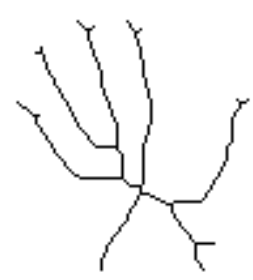

(b)

Fig. 1. (a) Original shape; (b) Shape skeleton

Considering the original set of vertices $V$, a new graph $G_{t}=\left(V, E_{t}\right)$, representing an intermediary stage in the graph evolution is yielded (Figure 2). Properties computed from this new version of the graph, such as the degree, can be computed and used to characterize its dynamics for different thresholds $t$, thus improving the characterization of the graph and, as a consequence, the original skeleton.

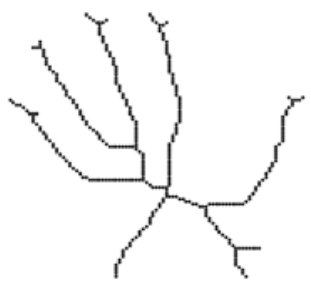

(a)

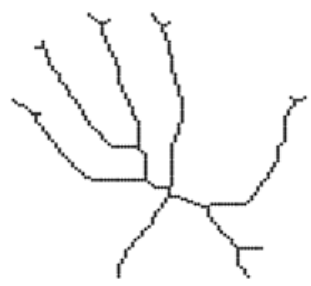

(b)

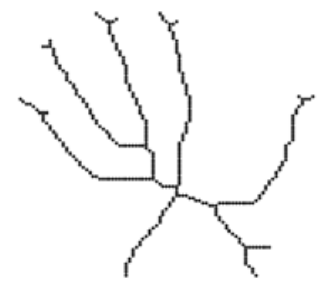

(c)

Fig. 2. Different stages in graph evolution. Edges in each graph are the only ones with weight smaller than a threshold $t$.

\section{Multi-scale Fractal Dimension of a Graph}

The vertex degree is one of the most used properties of a graph. However, literature provides various other measurements to characterize a graph. One of these measurements is the fractal dimension, a non-integer value related to the self-similarity and complexity of fractal objects 9 -11]. In fact, many real graphs are self-similar fractals 12 14].

The fractal dimension $d$ of a graph can be estimated from topological features, such as the graph degree. From the power law that exists between the graph degree $k$ and the distance $l$ used to compute this degree, the fractal dimension $d$ is defined as:

$$
k \approx l^{d}
$$

In our approach, the graph degree can be computed for different stages of the graph evolution $t$. Thus, the distance $l$ is the threshold $t$ used to compute such 


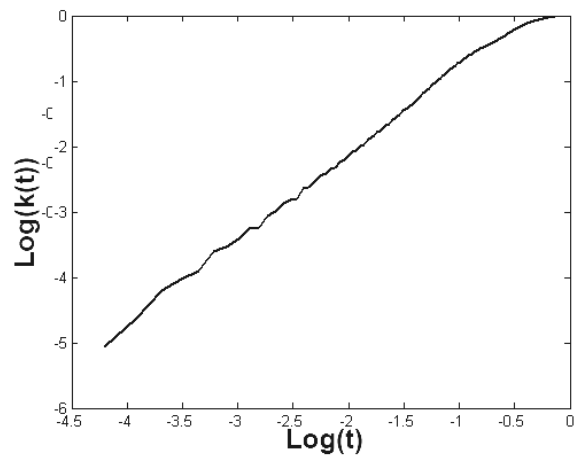

(a)

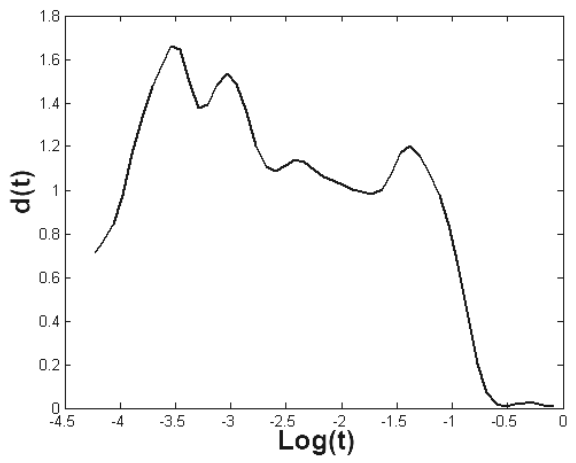

(b)

Fig. 3. (a) Log-log curve; (b) Multi-scale fractal dimension

measurement. Moreover, different degree based measurements can be considered, such as minimum degree $\operatorname{Min}\left(G_{t}\right)$, average degree $A v\left(G_{t}\right)$ and maximum degree $\operatorname{Max}\left(G_{t}\right)$ :

$$
\begin{aligned}
& \operatorname{Min}\left(G_{t}\right)=\frac{1}{N} \min _{i} \operatorname{deg}\left(v_{i}\right), \\
& \operatorname{Av}\left(G_{t}\right)=\frac{1}{N} \sum_{v_{i} \in V} \frac{\operatorname{deg}\left(v_{i}\right)}{|N|}, \\
& \operatorname{Max}\left(G_{t}\right)=\frac{1}{N} \max _{i} \operatorname{deg}\left(v_{i}\right),
\end{aligned}
$$

where $\operatorname{deg}\left(v_{i}\right)$ represents the degree of a vertice in the graph $G_{t}$. To avoid the influence of scale transformations in the degree measurement, its value is normalized into the interval $[0,1]$ by dividing the degree value by the total number of vertices in the network, $N$. Thus, the fractal dimension of a graph $G$ is defined as:

$$
d=\lim _{t \rightarrow 0} \frac{\log k(t)}{\log t},
$$

where $k(t)$ is one of the degree measurements considered.

To provide a more efficient and accurate description of the graph in terms of complexity, the Multi-Scale Fractal Dimension was also used. This method uses the derivative to exploit the infinitesimal limit of the linear interpolation, thus computing a curve which describes the changes in the graph complexity along the threshold used [15-17] (Figure 3). These changes in complexity are related to an irregular growing in the degree curve, which is most due to the presence of peculiarities in the graph and, as a consequence, in the original shape skeleton. Thus, the Multi-Scale Fractal Dimension is defined as:

$$
d(t)=\frac{d \log k(t)}{d \log t}
$$




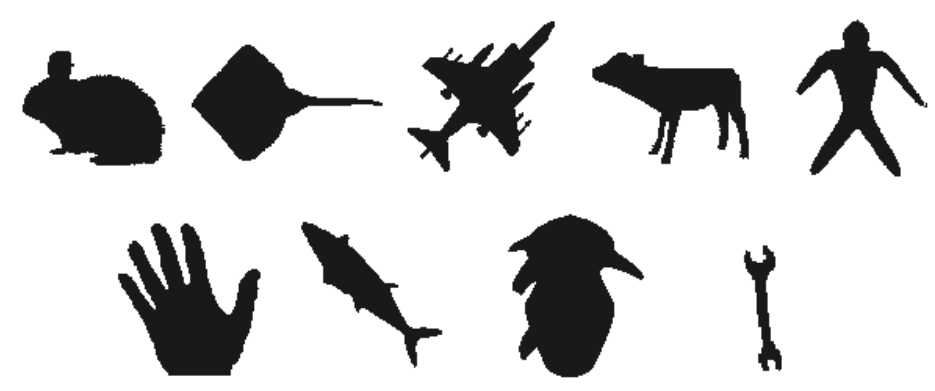

Fig. 4. Example of the artificial shapes used in the experiments

For Multi-Scale Fractal Dimension descriptors, we considered a feature vector containing the seven coefficients of the polynomial curve, computed by regression, that fits the derivative curve. Experiments have demonstrated that a degree higher does not improve the results [6].

\section{Experiments}

An experiment was set to evaluate the performance of the proposed approach. This experiment consists in to classify a set of 99 artificial shape images [18, 19]. These shapes are grouped in 9 different classes with 11 samples each. Each class represents a specific shape containing different variations in its structure, such as occlusion, articulation and missing parts (Figure 4).

A comparison with traditional shape analysis methods was also performed in order to provide a better evaluation of the proposed approach. Next, a brief description of each method considered for this comparison is presented:

Fourier descriptors: this approach uses the Fourier transform to compute the spectrum of the shape contour. The 20 most significant coefficients of the spectrum are used to compose a feature vector [16, 20].

Zernike moments: a feature vector is composed by considering the first 20 moments (order $n=0, \ldots, 7$ ), where each moment is the most significant magnitude computed from a set of orthogonal complex moments of the shape image [21].

Curvature descriptors: a curve is computed using the first and second derivatives of each component $x$ and $y$ of the shape contour. Points of maximum and minimum local of this curve describe changes in the shape contour orientation [22].

Bouligand-Minkowski: a curve able to describe how the shape complexity changes along the scale is computed from the influence area of the BouligandMinkowski fractal dimension method. In this approach, a feature vector is built by considering the 50 most meaningful points of the curve [3, 17].

Proposed and compared descriptors were evaluated using Linear Discriminant Analysis (LDA) in a leave-one-out cross-validation strategy [23, 24]. The LDA is a statistical method which searches a linear sub-space where data projection presents variance intra-classes larger than inter-classes. 


\section{$5 \quad$ Results}

Shape skeletons are able to represent a shape with reduced amount of information. Nevertheless, changes in the surface of shape may produce abrupt changes in its skeleton. Thus, a pruning method 25] is applied over the skeleton computed using the method by Choi et al. [26], so that, only the most significant ending branches remain in the skeleton.

As described in Section 3, different degree measurements may be used to estimate the Multi-scale fractal dimension and so the descriptors used in a shape classification experiment. Thus, it is necessary to evaluate the discrimination power of each different degree measurement, as also the combination of them. Table 1 expresses these results.

Table 1. Results for different complexity descriptors' sets and its combination for the artificial shapes database

Combined Degree Features

\begin{tabular}{|c|c|c|c|c|}
\hline Minimum & Average & Maximum & No of descriptors & Success rate $(\%)$ \\
\hline $\mathrm{X}$ & & & 7 & 75.76 \\
\hline & $\mathrm{X}$ & & 7 & 83.84 \\
\hline & & $\mathrm{X}$ & 7 & 78.79 \\
\hline $\mathrm{X}$ & $\mathrm{X}$ & & 14 & 85.86 \\
\hline $\mathrm{X}$ & & X & 14 & 86.87 \\
\hline & $\mathrm{X}$ & X & 14 & 91.92 \\
\hline $\mathrm{X}$ & $\mathrm{X}$ & X & 21 & 93.94 \\
\hline
\end{tabular}

Results indicated that the average degree presents the higher capacity to shape recognition, followed by the maximum degree and minimum degree, respectively. The average degree measurement that represents how the connectivity is distributed along the graph in a general way. Otherwise, the maximum degree and minimum degree are measurement related to the presence of saturated and nonsaturated vertices in the graph, respectively. Those vertices are the ones responsible for connecting different branches in the skeleton, junction nodes, and those which represent the end of a branch, end nodes. As expected, the combination of the three degree based measurements increases the success rate (93.94\%).

Table 2 shows the results achieved by each method in a comparative experiment. Our skeleton-based approach is compared to contour-based approaches, which are able to represent more efficiently all details present in a shape than the skeleton. Although the reduced amount of information, results of the proposed approach overcome the shape analysis methods considered in the experiment: Fourier descriptors, Zernike moments, Curvature descriptors and BouligandMinkowski. Moreover, the shape database includes shapes with different variations in their structure (such as, occlusion, articulation and missing parts), deformations that are a very common problem in shape acquisition, what contributes to validate the results yielded by proposed descriptors. 
Table 2. Classification performance of various shape descriptors for the artificial shapes database

\begin{tabular}{ccc}
\hline Shape descriptor & Shapes correctly classified Mean success rate (\%) \\
\hline Proposed Method & 93 & 93.94 \\
Fourier descriptors & 83 & 83.84 \\
Zernike moments & 91 & 91.92 \\
Curvature & 76 & 76.77 \\
Bouligand-Minkowski & 87 & 87.88 \\
\hline
\end{tabular}

\section{Conclusion}

This paper presented a novel approach for shape skeletons' characterization. There, a shape skeleton is represented and characterized as a graph in a dynamic evolution context. Degree based measurements are used to characterize this graph in terms of its complexity using the Multi-scale Fractal Dimension. Results demonstrated the potential of the approach in the discrimination of different shape patterns, including those presenting variations in their structure, overcoming traditional shape analysis, such as curvature, Fourier descriptors, Zernike moments and Bouligand-Minkowski.

\section{Acknowledgements}

A.R.B. acknowledges support from FAPESP (2006/54367-9). O.M.B. acknowledges support from CNPq (306628/2007-4).

\section{References}

1. Blum, H.: A transformation for extracting new descriptors of shape. In: Wathen-Dunn, W. (ed.) Models for the Perception of Speech and Visual Forms, pp. 362-380. MIT Press, Amsterdam (1967)

2. Blum, H., Nagel, R.: Shape description using weighted symmetric axis features. Pattern Recognition 10(3), 167-180 (1978)

3. da S. Torres, R., Falcão, A., da F. Costa, L.: A graph-based approach for multiscale shape analysis. Pattern Recognition 37, 1163-1174 (2003)

4. Wang, C., Cannon, D.J., Kumara, S.R.T., Guowen, L.: A skeleton and neural network-based approach for identifying cosmetic surface flaws. IEEE Transactions on Neural Networks 6(5), 1201-1211 (1995)

5. Zhu, X.: Shape recognition based on skeleton and support vector machines. In: Third International Conference on Intelligent Computing, pp. 1035-1043 (2007)

6. Backes, A.R., Bruno, O.M.: Shape classification using complex network and multiscale fractal dimension. Pattern Recognition Letters 31(1), 44-51 (2010)

7. Boccaletti, S., Latora, V., Moreno, Y., Chavez, M., Hwang, D.U.: Complex networks: Structure and dynamics. Physics Reports 424(4-5), 175-308 (2006)

8. da, F., Costa, L., Rodrigues, F.A., Travieso, G., Villas Boas, P.R.: Characterization of complex networks: A survey of measurements. Advances in Physics 56(1), $167-242(2007)$ 
9. Mandelbrot, B.: The fractal geometry of nature. Freeman \& Co., New York (2000)

10. Tricot, C.: Curves and Fractal Dimension. Springer, Heidelberg (1995)

11. Schroeder, M.: Fractals, Chaos, Power Laws: Minutes From an Infinite Paradise. W. H. Freeman, New York (1996)

12. Kim, J.S., Goh, K.I., Salvi, G., OH, E., Kahng, B., Kim, D.: Fractality in complex networks: critical and supercritical skeletons. Physical Review E 75, 16110 (2007)

13. Barabási, A.L., Albert, R.: Emergence of scaling in random networks. Science 286(5439), 509-512 (1999)

14. Feder, J.: Fractals. Plenum, New York (1988)

15. Emerson, C.W., Lam, N.N., Quattrochi, D.A.: Multi-scale fractal analysis of image texture and patterns. Photogrammetric Engineering and Remote Sensing 65(1), 51-62 (1999)

16. Gonzalez, R.C., Woods, R.E.: Digital Image Processing, 2nd edn. Prentic-Hall, New Jersey (2002)

17. Plotze, R.O., Padua, J.G., Falvo, M., Vieira, M.L.C., Oliveira, G.C.X., Bruno, O.M.: Leaf shape analysis by the multiscale minkowski fractal dimension, a new morphometric method: a study in passiflora (passifloraceae). Canadian Journal of Botany-Revue Canadienne de Botanique 83, 287-301 (2005)

18. Sebastian, T.B., Klein, P.N., Kimia, B.B.: Recognition of shapes by editing their shock graphs. IEEE Trans. Pattern Analysis and Machine Intelligence 26(5), 550-571 (2004)

19. Sharvit, D., Chan, J., Tek, H., Kimia, B.B.: Symmetry-based indexing of image databases. Journal of Visual Communication and Image Representation 9(4), 366-380 (1998)

20. Osowski, S., Nghia, D.D.: Fourier and wavelet descriptors for shape recognition using neural networks - a comparative study. Pattern Recognition 35(9), 1949-1957 (2002)

21. Zhenjiang, M.: Zernike moment-based image shape analysis and its application. Pattern Recognition Letters 21(2), 169-177 (2000)

22. Wu, W.Y., Wang, M.J.: On detecting the dominant points by the curvature-based polygonal approximation. CVGIP: Graphical Models Image Process 55, 79-88 (1993)

23. Everitt, B.S., Dunn, G.: Applied Multivariate Analysis, 2nd edn. Arnold (2001)

24. Fukunaga, K.: Introduction to Statistical Pattern Recognition, 2nd edn. Academic Press, London (1990)

25. Bai, X., Latecki, L.J., Liu, W.Y.: Skeleton pruning by contour partitioning with discrete curve evolution. IEEE Trans. Pattern Analysis and Machine Intelligence 29(3), 449-462 (2007)

26. Choi, W.P., Lam, K.M., Siu, W.C.: Extraction of the euclidean skeleton based on a connectivity criterion. Pattern Recognition 36(3), 721-729 (2003) 